\title{
ESTUDO QUÍMICO DE EFLORESCÊNCIAS SALINAS DO SÍTIO ARQUEOLÓGICO TOCA EXÚ DO JURUBEBA DO PARQUE NACIONAL SERRA DA CAPIVARA, PIAUÍ, BRASIL
}

\author{
Benedito Batista Farias Filho ${ }^{a}$,*, Maria Conceição S. Meneses Lage e Rássius Alexandre Medeiros Lima ${ }^{\mathrm{a}}$ \\ aDepartamento de Química, Universidade Federal do Piauí, 64049-550 Teresina - PI, Brasil

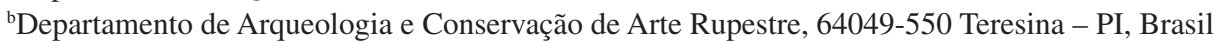

Recebido em 07/02/2017; aceito em 04/05/2017; publicado na web em 03/08/2017

\begin{abstract}
CHEMICAL STUDY OF SALTS EFFLORESCENCES OF THE ARCHAEOLOGICAL SITE TOCA EXÚ DO JURUBEBA OF THE NATIONAL PARK SERRA DA CAPIVARA, PIAUÍ, BRAZIL. Toca Exú do Jurubeba is part of a set of archaeological sites located in the Serra da Capivara National Park (Piauí, Brazil), a region of great world interest due to its archaeological potential, especially rock art. However, rock art is constantly threatened by both natural and anthropic factors. Among the natural salts efflorescences that are deposited on the painted graphics often even concealing them. The present study consisted of the spectroscopic analysis of different saline efflorescence samples of the Serra da Capivara National Park, using examination techniques (optical microscopy and Scanning Electron Microscopy) and analysis (Fourier Transform Infrared and Laser Induced Breakdown Spectroscopy). The analysis allowed to identify at least four different compositions of the samples as a mixture of hydrated calcium oxalate $\left(\mathrm{CaC}_{2} \mathrm{O}_{4} \mathrm{H}_{2} \mathrm{O}\right)$ with kaolinite $\left(\mathrm{Al}_{2} \mathrm{SiO}_{3}(\mathrm{OH})_{4}\right)$, gypsum $\left(\mathrm{CaSO}_{4} 2 \mathrm{H}_{2} \mathrm{O}\right)$, tetrahydrate calcium nitrate $\left(\mathrm{Ca}\left(\mathrm{NO}_{3}\right)_{2} 4 \mathrm{H}_{2} \mathrm{O}\right)$ and aluminosilicates in the state of fossilization. The obtained results open the way for the conservation of rock art, initially in the archaeological site under study and can serve as basis for other interventions that may be developed in the region.
\end{abstract}

Keywords: spectroscopy; salt efflorescence; rock art; conservation science.

\section{INTRODUÇÃo}

Os sítios arqueológicos contêm informações sobre grupos humanos que habitaram no passado. Estas informações são extraídas de vestígios que conseguiram chegar até os dias atuais, cuja análise permite reconstituir o cenário vivido pelo homem pré-histórico. Dentre estes vestígios, a arte rupestre permite uma visão mais detalhista sobre o modo de vida de comunidades pretéritas, pois trata-se de um sistema de comunicação visual elaborado para expressar o cotidiano, o qual inclui rituais religiosos, práticas domésticas, entre outros. ${ }^{1}$ No entanto, toda essa riqueza arqueológica está ameaçada por vários problemas de conservação, causadas por fatores naturais e/ou antrópicos.

As pinturas rupestres estão sujeitas a processos de degradação acelerada, o que inclui os problemas relacionados à formação de depósitos minerais nas paredes rochosas. Esses depósitos, também chamados de eflorescências salinas, são agregados cristalinos de sais que apresentam forma e composição química variada e que se depositam sobre a superfície de uma estrutura rochosa. ${ }^{2,3}$ São materiais também encontrados em estruturas da construção civil, monumentos históricos e obras de arte..$^{4-7}$

A origem das eflorescências salinas são as mais diversas, mas em geral, são formadas pela migração de sais solúveis e insolúveis, presentes na região interna da rocha e que são carreadas pela água para a sua superfície através de sua rede de capilaridade. ${ }^{8,9}$ Esses sais, quando expostos à temperatura do ambiente externo, cristalizam-se, formando uma estrutura que pode se apresentar de diferentes colorações. Além disso, quando na superfície rochosa podem reagir com materiais do ambiente externo, como as substâncias de plantas, o que justifica a diversidade na sua composição química. ${ }^{9-11}$

Técnicas espectroscópicas têm sido utilizadas para caracterização química de eflorescências salinas, sobretudo em obras de arte e empregam a Microscopia Eletrônica de Varredura acoplada à Espectroscopia de Energia Dispersiva (MEV/EDS), ${ }^{12-14}$ a Difração de

*e-mail: benedito.filho@hotmail.com
Raios X (DRX) ${ }^{15,16}$ e a espectroscopia vibracional, ${ }^{17}$ além da análise térmica (DSC/TGA/DTA). ${ }^{18,19} \mathrm{O}$ uso dessas técnicas analíticas para a caracterização das eflorescências salinas direciona os restauradores e/ou conservadores a proporem procedimentos de intervenção a ser executados seja em obras de artes ou pinturas rupestres para garantir que tais patrimônios culturais cheguem a gerações futuras.

O Parque Nacional Serra da Capivara, localizado no estado do Piauí (Brasil), tombado pela UNESCO como patrimônio cultural da humanidade em 1991, possui grande quantidade de sítios de arte rupestre em toda sua área de conservação ambiental. ${ }^{1,20,21}$ As pinturas rupestres têm sido alvo de estudo desde a década de 70 e têm revelado que a sua composição química são óxidos minerais de ferro como a hematita, a goetita, a magnetita, entre outras. ${ }^{22}$ Trata-se de uma arte pré-histórica que apresenta riqueza de detalhes em seus desenhos, preenchimentos, e são elaboradas em diversas cores (vermelho, amarelo, branco, preto, azul, cinza), preparadas com matéria-prima disponível no local.

Dentre os sítios arqueológicos do Parque Nacional Serra da Capivara encontra-se a Toca Exú do Jurubeba (Figura 1), localizado no município de Coronel José Dias nas coordenadas geográficas UTM L 764957 e UTM N 9019386.

O sítio arqueológico em estudo é um abrigo sob rocha arenítica com dimensão de 20 metros de comprimento e 5 metros de altura e apresenta grande quantidade de pinturas rupestres em diversas tonalidades de vermelho. No entanto, o sítio está ameaçado por problemas de conservação, sobretudo de origem natural, devido à presença de sais brancos, que escorrem sobre o paredão rochoso e que acaba por deteriorar o seu valioso patrimônio artístico, cultural e histórico.

Assim, o objetivo do presente trabalho foi o de analisar a composição química de eflorescências salinas presentes no sitio arqueológico Toca Exú do Jurubeba, localizado no Parque Nacional Serra da Capivara, visando desacelerar o grau de degradação das pinturas rupestres através de ações preventivas e interventivas de conservação. Para isso, utilizou-se a espectroscopia no infravermelho com Transformada de Fourier (FTIR) e a espectroscopia de emissão em 

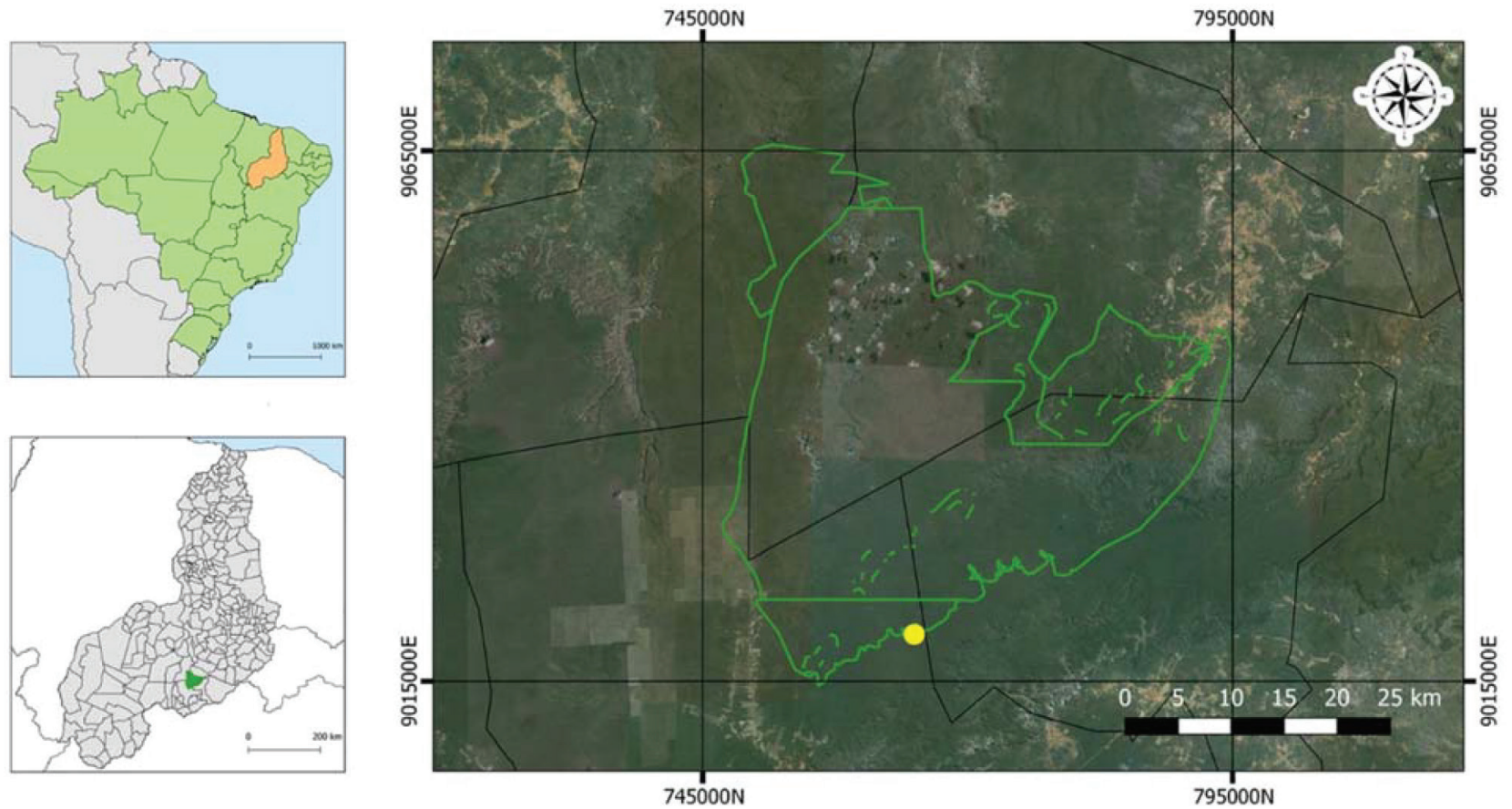

Legenda

Sítio Arqueológico Toca Exú do Jurubeba

Parque Nacional Serra da Capivara

Figura 1. Localização geográfica do sítio arqueológico Toca Exú do Jurubeba

plasma induzido por laser (LIBS), além dos exames sob microscopia óptica (MO) e microscopia eletrônica de varredura (MEV). A utilização dessas técnicas de análises permite avaliar as suas potencialidades para esses tipos de amostra, cujo conhecimento é de grande interesse para ciência da conservação em arqueologia.

\section{PARTE EXPERIMENTAL}

\section{Amostras}

Foram coletadas 6 micro-amostras de eflorescências salinas (SJ04.04, SJ04.06, SJ04.07, SJ05.04, SJ05.05 e SJ05.06) presentes em pontos diferentes da parede rochosa do citado sítio arqueológico Toca Exú do Jurubeba.

As amostras foram retiradas de áreas distantes das pinturas rupestres priorizando os locais onde o estado de degradação estivesse mais avançado e tomando o cuidado para que fosse realizada uma amostragem representativa do material. Todo o processo de coleta das amostras foi realizado obedecendo às normas e regras determinadas pelas cartas patrimoniais propostas pelo ICOMOS. ${ }^{23}$

\section{Caracterização físico-química das amostras}

Observação sob microscópio óptico

Uma pequena porção das amostras de eflorescência salina foi colocada sobre uma lâmina de vidro e examinada sob a ocular de um microscópio portátil (ProScope HR CSI) com conexão USB ao computador. As imagens foram obtidas utilizando uma lente (Scalar) de aumento de 50x realizando uma observação minuciosa do depósito salino.

Microscópio Eletrônico de Varredura

$\mathrm{O}$ estudo morfológico das amostras foi realizado em um microscópio eletrônico de varredura (Fei Company, Quanta Feg 250) operando com tensão de $20 \mathrm{kV}$. Previamente as amostras foram fixadas em fita adesiva de carbono dupla fase e, posteriormente, realizada a metalização com ouro. As leituras foram obtidas em aumentos de 5000 a 10000 vezes.

\section{Infravermelho com Transformada de Fourier}

As medidas por infravermelho (Agilent Technology, CARY 630 FTIR) foram realizadas no modo ATR (reflectância total atenuada), colocando-se pequenas quantidades das amostras sobre o porta-amostra do instrumento e prensadas sobre um cristal de diamante. As medidas foram realizadas no intervalo de 4000 a $400 \mathrm{~cm}^{-1} \mathrm{com}$ resolução espectral de $4 \mathrm{~cm}^{-1}$ e utilizando 64 varreduras.

Espectroscopia de emissão óptica em plasma induzido por laser

As análises elementares foram realizadas utilizando um equipamento LIBS constituído por um policromador echelle (Mechelle 5000, Andor Technology), laser pulsado Nd:YAG $(20 \mathrm{~Hz}, 1064 \mathrm{~nm}$, FWHM 5,2 ns, e energia máxima do pulso de $110 \mathrm{~mJ}$ ) e detector ICCD (iStar DH734, Andor Technology). A superfície de uma amostra de pigmento rupestre contém superfície irregular, fazendo com que, para cada medida, a localização exata do plasma a ser formado seja alterada. Para minimizar esse efeito, empregou-se duas ponteiras laser de tal maneira que estas emergissem a uma certa distância da lente. A plataforma na qual a amostra se encontra é removível, e para cada amostra analisada, as ponteiras eram alinhadas até que as radiações laser fossem interceptadas. As medidas foram realizadas empregando energia do laser de $20 \mathrm{~mJ} /$ pulso, tempo de delay de $500 \mathrm{~ns}$ e tempo de integração de $1 \mu \mathrm{s}$. Os sinais analíticos obtidos foram identificados utilizando-se a base de dados do NIST Atomic Spectra library. As medidas foram realizadas em triplicatas, acumulando três pulsos em locais distintos ao longo da superfície da amostra para garantir uma medida representativa. 

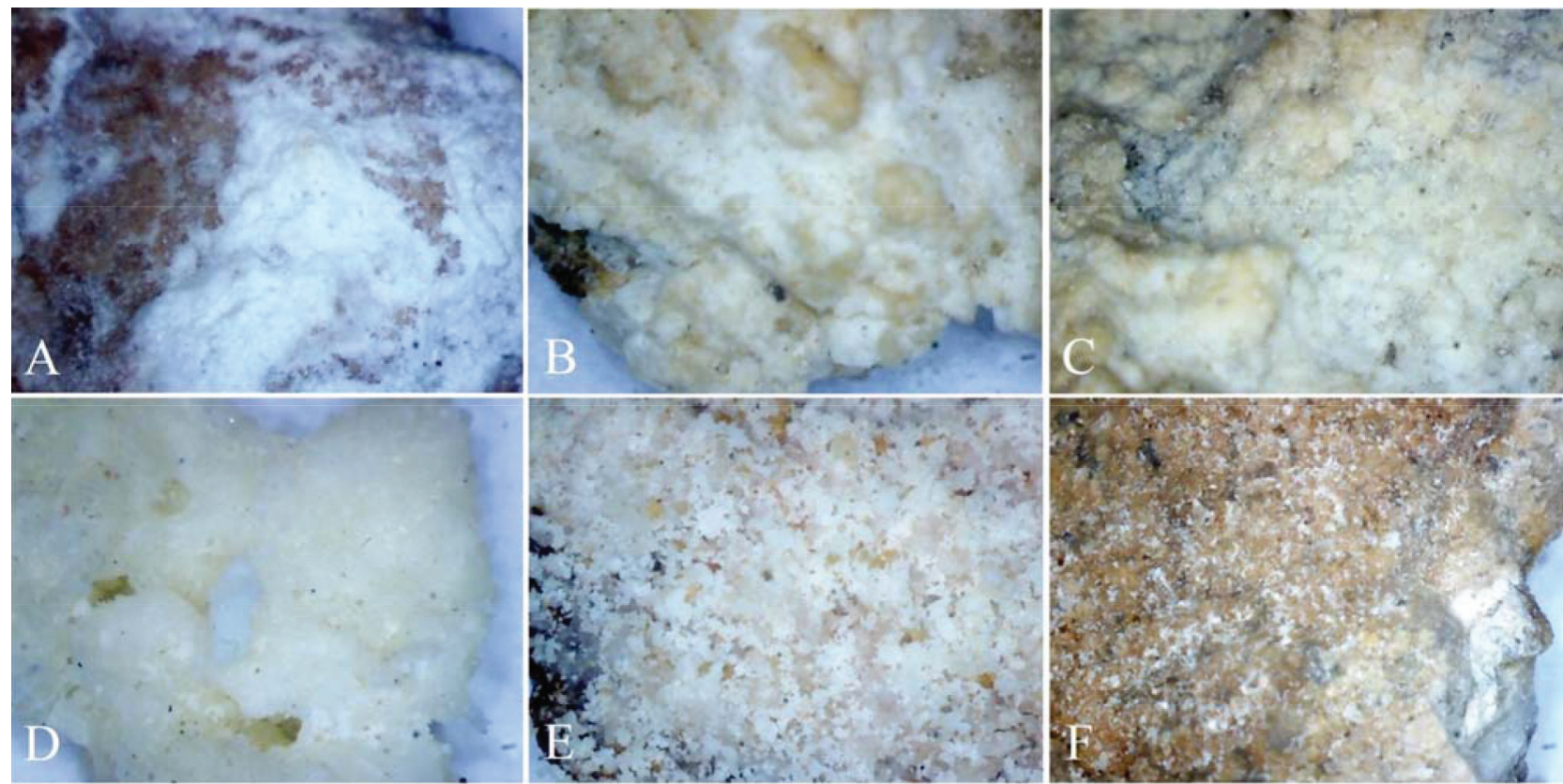

Figura 2. Exames sob microscopia óptica das amostras de eflorescência salina (A) SJ04.04, (B) SJ04.06, (C) SJ04.07, (D) SJ05.04, (E) SJ05.05 e (F) SJ05.06 com detalhes em aumento de $50 x$

\section{RESULTADOS E DISCUSSÃO}

As amostras de eflorescências salinas foram submetidas a métodos de exames e análises químicas para a sua caracterização e os resultados servirem de base para etapas e trabalhos de conservação do sítio arqueológico Toca Exú do Jurubeba presente em uma área de grande importância mundial, o Parque Nacional Serra da Capivara. Foram elegidas as técnicas de exames mais comumente utilizadas em amostras de interesse arqueológico, como a microscopia óptica e a Microscopia Eletrônica de Varredura, que fornecem dados sobre a morfologia do material analisado. As técnicas espectroscópicas LIBS e FTIR foram escolhidas para este trabalho por ambas fornecerem sinais analíticos satisfatórios para complementação das análises, e o atrativo para medidas arqueológicas, que é a possibilidade de serem utilizadas sem nenhum preparo de amostra.

Os exames sob microscópio óptico (Figura 2) permitiram visualizar informações das eflorescências como coloração, tamanho e forma de partículas, bem como sua aderência ao paredão rochoso, características não disponíveis a olho nu. Além disso, o presente estudo utilizando os exames microscópios foram fundamentais para interpretação inequívoca das análises por LIBS e FTIR, uma vez que este tipo de amostra possui natureza heterogênea.

A observação sob microscopia óptica das amostras permitiu revelar que os sais de eflorescências salinas já se encontram distribuídas sobre a superfície rochosa, embora ainda de forma não-uniforme. Ao mesmo tempo, foi possível observar que cada amostra coletada possui características distintas, principalmente em relação à aderência na rocha. Em especial nas amostras B e C os sais se encontram incrustados sobre a superfície rochosa, com dificuldades de desagregá-las, o que leva a inferir que está em estado de fossilização, enquanto que para as demais amostras (A, D, E e F) os sais estão em pequenas porções, fáceis de serem removidas da matriz rochosa. A amostra $\mathrm{E}$ apresenta uma característica distinta em relação às demais, com a forma de pequenas agulhas ou fibras longas distribuídas na rocha.

As micrografias das amostras de eflorescência salina obtidas por MEV (Figura 3), utilizando o aumento de 10000 vezes, permitiram observar diferentes aspectos morfológicos.
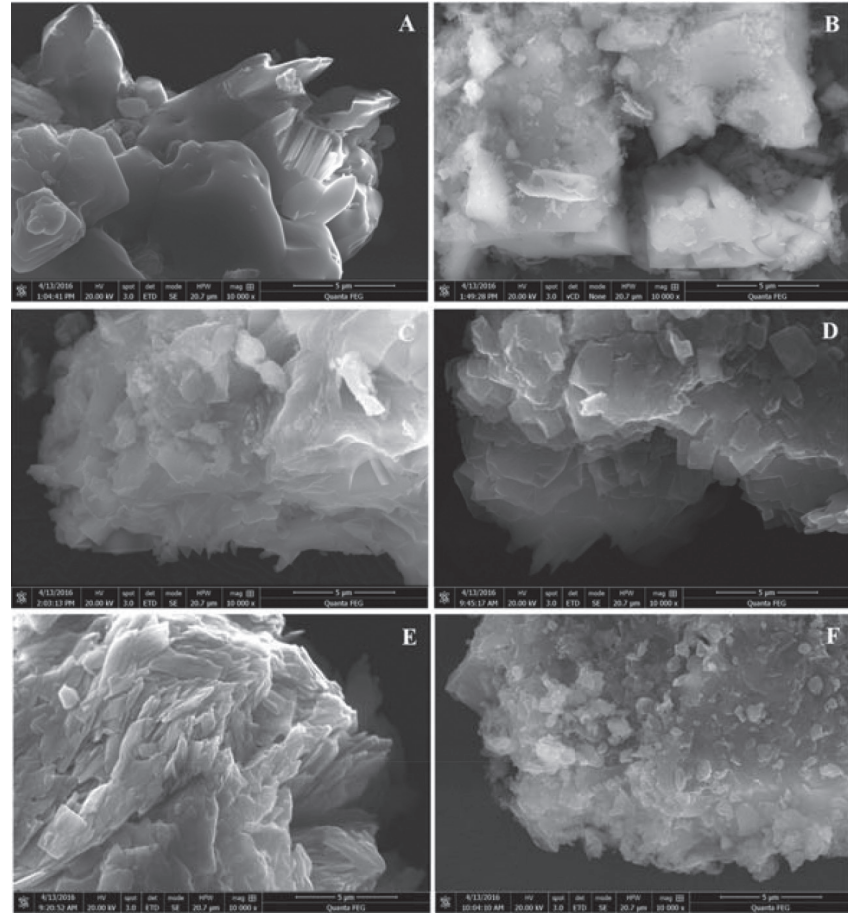

Figura 3. Micrografias das amostras de eflorescência salina (A) SJ04.04, (B) SJ04.06, (C) SJ04.07, (D) SJ05.04, (E) SJ05.05 e (F) SJ05.06 com detalhes em aumento de $10.000 x$

A amostra SJ04.04 revela uma morfologia de estrutura densa e compacta e muitos pontos em formato de agulha. Contrariamente, as amostras SJ04.06 (Figura 3B), SJ04.07 (Figura 3C) e SJ05.06 (Figura 3F) apresentam no formato de uma massa densa, bem como alguns grãos de formato retangular e outros arredondados ao longo de sua superfície.

É possível observar também nas amostras SJ05.04 (Figura 3D) e SJ05.05 (Figura 3E) um aspecto morfológico bastante semelhante na forma de várias estruturas planas retangulares que se sobrepõem e formam camadas laminares. 
Embora poucos detalhes foram possíveis de serem observados nas micrografias das amostras, conhecer a morfologia através de técnicas de exame permitiu fornecer informações de materiais que venham estar agregados aos depósitos minerais e com isso dificultar a interpretação dos resultados baseados na análise qualitativa.

A Figura 4 mostra os resultados da análise qualitativa das amostras com os principais sinais de emissão e seus respectivos elementos químicos que foram obtidos, realizando um exame minucioso de toda a faixa espectral obtidos por LIBS.

Foram selecionadas apenas as linhas de emissão mais intensas para realizar a caracterização química da amostra. A linha de emissão de maior intensidade para todas as amostras refere-se ao Ca II em $396,8 \mathrm{~nm}$ e presentes também em $315,91 \mathrm{~nm}, 422,70 \mathrm{~nm}, 430,24 \mathrm{~nm}$, $445,50 \mathrm{~nm}, 534,96$ e $616,26 \mathrm{~nm}$. Esse resultado pode ser responsável, em parte, pela composição química-mineralógica das eflorescências salinas, resultado da reação dos ânions solúveis que migram do interior da rocha (sulfatos, carbonatos e outros), bem como de substâncias químicas, como o ácido húmico (liberadas pelas raízes de plantas), que formam os agregados salinos.

A Figura 4 mostra ainda outros componentes presentes nas amostras, uns de caráter majoritários e outros em nível de traços. Foram detectadas claramente as linhas de emissão atômica do $\mathrm{Na}$ I (dupleto em 588.99 e $589.59 \mathrm{~nm})$, Si I (288, $17 \mathrm{~nm})$, Al I (309,31 $\mathrm{nm})$ e Fe II $(573,73 \mathrm{~nm} ; 404,59 \mathrm{~nm}$ e 445,49 nm)). Esses resultados estão em concordância com a composição de uma infinidade de eflorescências salinas já estudadas, oriundas de fontes da construção civil ${ }^{4}$ arquitetônica histórica, ${ }^{5}$ obras de arte e arqueológicas. ${ }^{5,6}$ Além disso, os elementos como Si e Al podem estar associados diretamente à composição do suporte rochoso que são também compostos de aluminossilicatos. Elementos como $\mathrm{Mg}$ I (279,56 nm e 383,20 nm), Ti II (336,11 nm; 368,52 nm e 518,39 nm), K I (766,49 nm), Mn I
(714,88 e 612,27 nm), Li I (670,73 nm) e Ba I (493,41 nm) podem estar associados a impurezas contidas nas amostras, uma vez que se trata de um sítio arqueológico a céu aberto e, por isso, as ações ambientais como o vento, que arrastam materiais até as paredes rochosas e acabam se depositando, são bastante atuantes.

A caracterização química utilizando a técnica LIBS foi fundamental para realização do levantamento inicial dos principais elementos químicos presentes nas amostras. Assim, para a caracterização completa dos depósitos minerais foi elegida a técnica FTIR, que em conjunto com o LIBS forneceu a caracterização químico-mineralógica completa da amostra, permitindo assim os trabalhos de conservação de arte rupestre.

Na Figura 5 são apresentados os espectros FTIR das amostras de eflorescências salinas coletadas no paredão rochoso do sítio arqueológico Toca do Exú do Jurubeba. As análises espectrais FTIR revelaram que pelo menos três compostos fazem parte do conjunto de amostras analisadas e que algumas delas já estão em estado de silicificação, devido à presença marcante de modos vibracionais do $\mathrm{SiO}_{2}$ ou da mistura com fragmentos rochosos que vêm aderido aos depósitos de eflorescências salinas.

A amostra SJ.05.06 apresenta bandas de estiramento de Si-O em 1032 e $1008 \mathrm{~cm}^{-1}$, em $913 \mathrm{~cm}^{-1}$ é associada ao modo vibracional de deformação do Al-OH e em $534 \mathrm{~cm}^{-1}$ é ligada ao modo de deformação do Si-O-Al. ${ }^{24,25}$ Além dessas bandas foi possível observar em 1619 $\mathrm{cm}^{-1}$ o modo vibracional de estiramento da carbonila $(\mathrm{C}=\mathrm{O})$, bem como em $1339 \mathrm{~cm}^{-1}$ que é devido ao estiramento simétrico C-O-C, e a banda em $780 \mathrm{~cm}^{-1}$ que é da soma dos modos vibracionais de estiramento O-C-O e de deformação de C-O, $[v(\mathrm{O}-\mathrm{C}-\mathrm{O})+\delta(\mathrm{C}-\mathrm{O})]{ }^{26}$ A banda larga em aproximadamente $3400 \mathrm{~cm}^{-1}$ se deve ao modo de estiramento da hidroxila de água. ${ }^{26}$ Essas informações permitem identificar inequivocamente que tal amostra é composta de whewellita
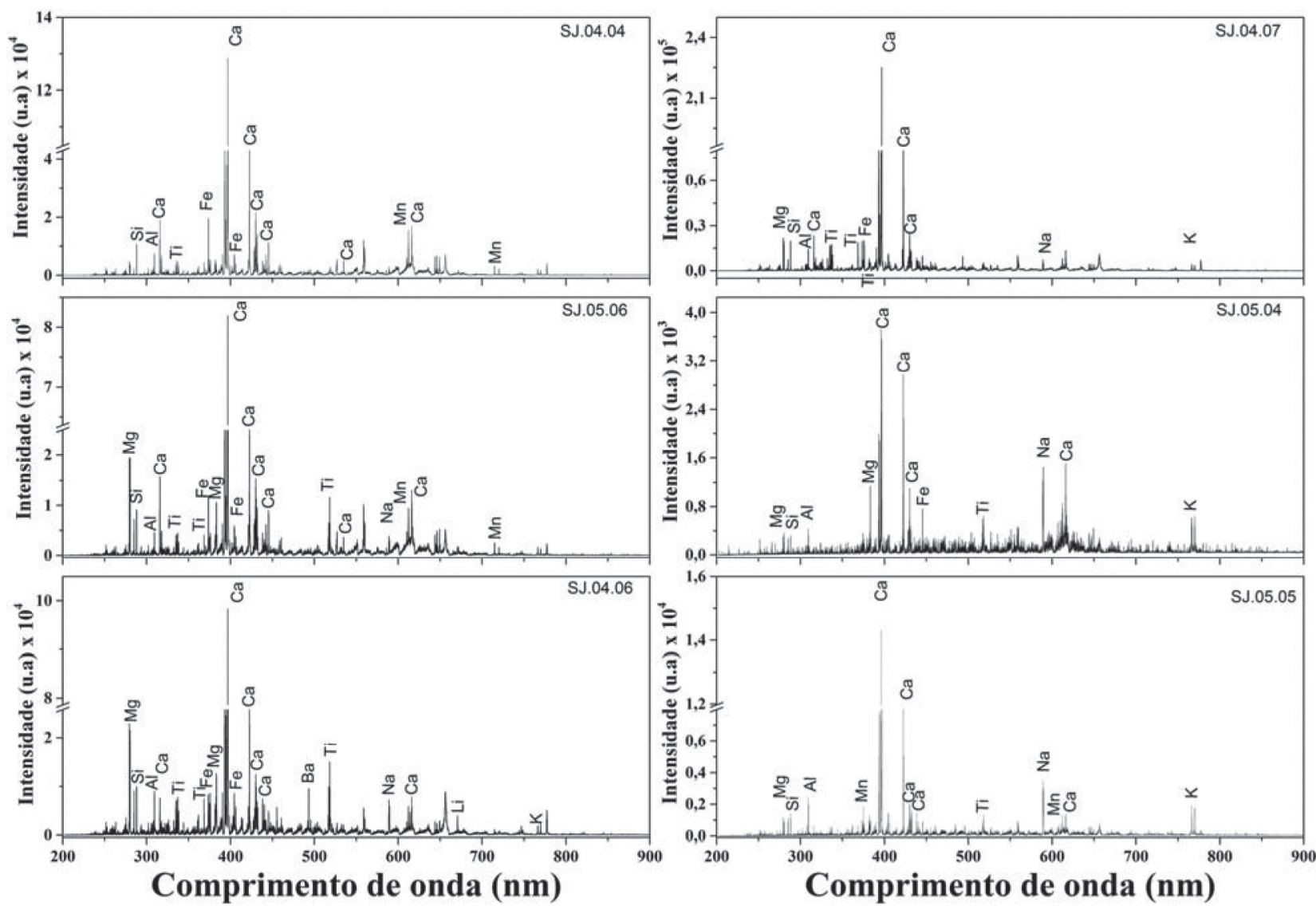

Figura 4. Espectro LIBS das amostras de eflorescência salina 

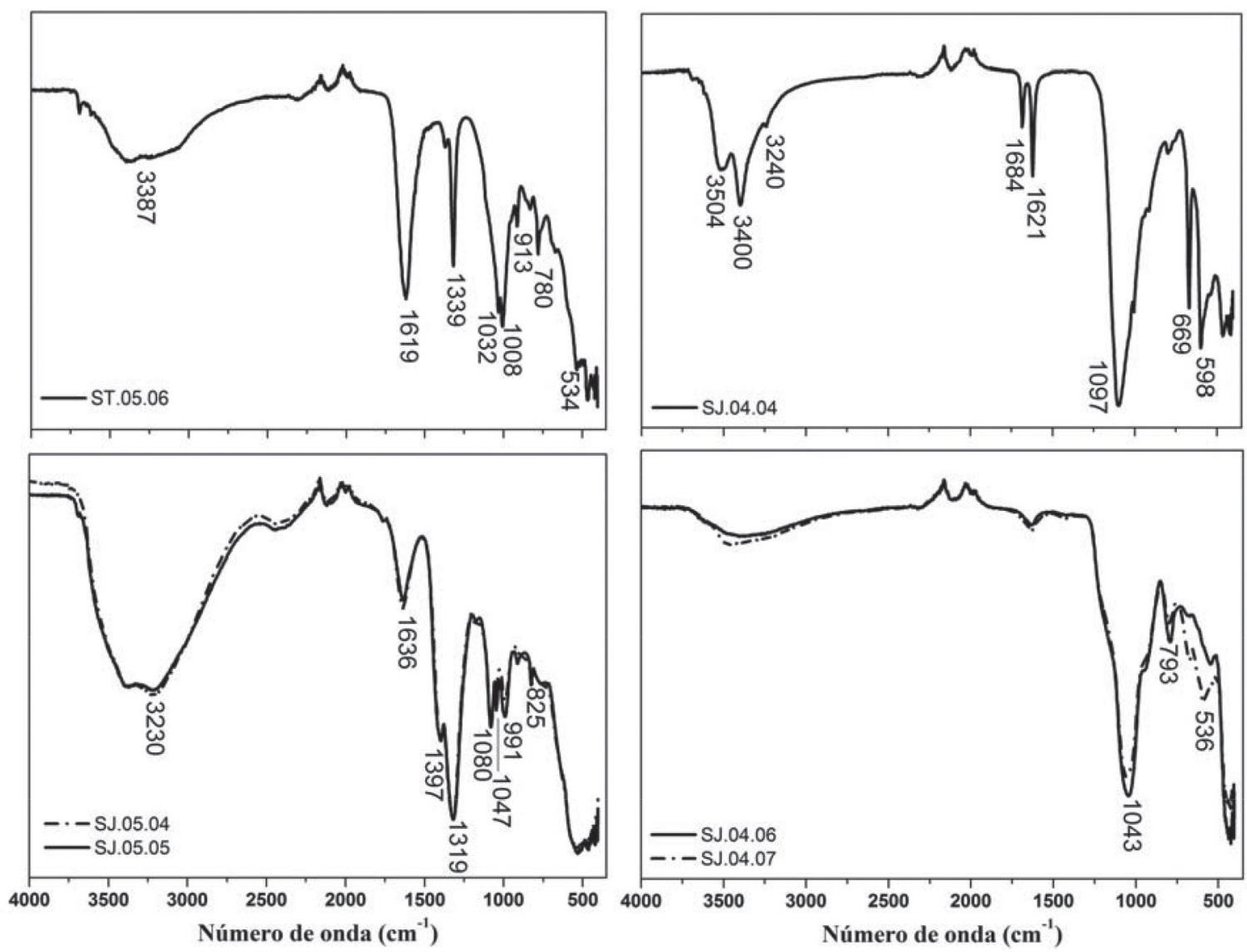

Figura 5. Espectros de absorção no infravermelho das amostras de eflorescências salinas

$\left(\mathrm{CaC}_{2} \mathrm{O}_{4} \cdot \mathrm{H}_{2} \mathrm{O}\right)$ com a presença de caolinita $\left[\mathrm{Al}_{2} \mathrm{Si}_{2} \mathrm{O}_{5}(\mathrm{OH})_{4}\right]$. O mecanismo de formação do oxalato de cálcio em rochas já é conhecido na literatura e ocorre pela reação do ácido oxálico (liberado por líquens, cogumelos e plantas radiculares) e as substâncias presentes na rocha, cujos produtos finais são oxalatos de cálcio em diferentes formas de hidratação..$^{9,10}$

A amostra SJ.04.04 apresentou bandas vibracionais na região de $3000 \mathrm{~cm}^{-1}\left(3504,3400\right.$ e $\left.3240 \mathrm{~cm}^{-1}\right)$, além de $598 \mathrm{~cm}^{-1}$ que é atribuída ao estiramento de $\mathrm{OH}$ de água. Os modos vibracionais de deformação de $\mathrm{OH}$ ainda apareceram como bandas bem definidas em 1684 e 1621 $\mathrm{cm}^{-1}$. As assinaturas das bandas em 1097 e $669 \mathrm{~cm}^{-1}$ são atribuídas aos modos de estiramento de $\mathrm{S}-\mathrm{O}$ de íons sulfatos $\left(\mathrm{SO}_{4}{ }^{2-}\right){ }^{27}$ Todas as características espectrais, bem como a análise prévia da composição elementar, permitem inferir que a eflorescência salina em estudo se trata do sulfato de cálcio bihidratado $\left(\mathrm{CaSO}_{4} \cdot 2 \mathrm{H}_{2} \mathrm{O}\right)$.

As amostras SJ.04.06 e SJ.04.07 tiveram perfis espectrais bastante semelhantes e apresentaram bandas características da caolinita, cujos modos de vibração em $1043 \mathrm{~cm}^{-1}$ são atribuídos ao estiramento de $\mathrm{Si}-\mathrm{O}$, em $793 \mathrm{~cm}^{-1}$ a banda de vibração é característica de estiramento $\mathrm{Si}$, e, em $536 \mathrm{~cm}^{-1}$, corresponde à vibração de deformação de Si-OA1. ${ }^{25}$ Para estas amostras foi detectada apenas bandas de vibração de aluminossilicatos, o que indica que os sais se encontram em estado de silicificação. Essas amostras, como mostrado no exame sob microscópio (Figura 2), encontram-se agregadas ao suporte rochoso, o que indica que esse processo de silicificação ocorreu devido ao longo tempo de exposição às características naturais, por exemplo a radiação solar.

Por fim, as amostras SJ.05.04 e SJ.05.05 também apresentaram composição química semelhante em comparação com as principais bandas de vibração no infravermelho. Pode-se observar uma banda larga e intensa ao redor de $3230 \mathrm{~cm}^{-1}$ e uma banda bem definida em
$1636 \mathrm{~cm}^{-1}$ que pode ser atribuída respectivamente ao modo de estiramento e deformação da hidroxila da água. ${ }^{27-29}$ As demais bandas levam a entender que se trata de um sal a base de nitrato, confirmado pela presença da banda de estiramento do $\mathrm{N}-\mathrm{O}$ em $1397 \mathrm{~cm}^{-1}$ e em 825 $\mathrm{cm}^{-1}$, que se refere ao estiramento $\mathrm{N}=\mathrm{O}^{29,30}$ Além disso, outras bandas de absorção do N-O do íon nitrato podem ser observadas em 1047 e em $1319 \mathrm{~cm}^{-1}$, que equivalem ao estiramento simétrico e assimétrico respectivamente. ${ }^{31}$ Assim, essas informações em conjunto sugerem que o depósito salino para as amostras SJ.05.0 e SJ.05.05 se trata do sal de $\mathrm{Ca}\left(\mathrm{NO}_{3}\right)_{2} \cdot 4 \mathrm{H}_{2} \mathrm{O}$.

A região do Parque Nacional Serra da Capivara contém mais de 1200 sítios arqueológicos e apesar de ser uma extensa área territorial o controle dos problemas de conservação vem sendo monitorados há décadas por uma equipe multidisciplinar encarregada de resolver problemas de conservação para a manutenção dos sítios arqueológicos. No entanto, alguns problemas pontuais, como as eflorescências salinas, ainda são as mais difíceis de serem controladas uma vez que são processos produzidos pela própria rocha por um processo natural. Assim, considerando que cada sítio arqueológico é um bem único dentro do contexto cultural e histórico, a produção das eflorescências salinas dentro de um mesmo sítio arqueológico pode apresentar características químicas distintas, como foi o caso do sítio em estudo.

Uma vez que as amostras foram caracterizadas físico-quimicamente, percebe-se a existência de quatro grupos distintos que auxiliarão as etapas de conservação e que serão utilizadas dentro do sítio em estudo, aquelas em que se encontram em estado de fossilização e aquelas que ainda mantêm todas as suas características químicas originais.

Para as etapas de intervenção arqueológica do sítio Exú do Jurubeba, propõe-se inicialmente a utilização de retiradas mecânicas, sobretudo do grupo em que a sua composição química se trata de um 
material já em estado de silicificação, característica das amostras SJ.04.06 e SJ.04.07. Essa etapa é necessária porque, como apontado nos exames sob microscópio, apesar de algumas das amostras se encontrarem aderidas na rocha, elas se acumulam formando espessas camadas de sais, que podem ser removidas antes de chegarem à superfície rochosa.

Uma vez concluída a limpeza mecânica, a segunda etapa consistirá de um tratamento químico, principalmente para o grupo das amostras SJ.05.06, SJ.04.04, SJ.05.04 e SJ.05.05 no sentido de solubilizar todo o material, uma vez que elas preservaram sua estrutura química. Vale a pena ressaltar todo o cuidado na escolha dos reagentes e a forma como utilizá-los nos paredões rochosos sem que venham a provocar qualquer dano às pinturas rupestres presentes no sítio.

\section{CONCLUSÃO}

A análise por LIBS em conjunto com a FTIR, bem como os exames morfológicos por microscopia ótica e microscopia eletrônica de varredura foram suficientes para realizar a caracterização completa das eflorescências salinas presentes no sítio arqueológico Toca Exú da Jurubeba. As técnicas mostraram potencialidades para serem aplicadas em amostras dessa natureza por dispensar o preparo de amostras e fornecer informações sobre a natureza química dos depósitos minerais, importantes para os trabalhos de conservação de arte rupestre. Nós reportamos que as eflorescências salinas são compostas de diferentes sais como uma mistura de oxalato de cálcio hidratado com caolinita, sulfato de cálcio e nitrato de cálcio, além de um depósito material em estado de silicificação.

Os resultados obtidos para as amostras em estudo servirão de base para trabalhos futuros de intervenção de conservação no sítio arqueológico Toca Exú do Jurubeba, mas também poderão se estender como base para outros sítios arqueológicos da região do Parque Nacional Serra da Capivara, bem como em sítios arqueológicos que também passam pelo mesmo problema de conservação.

\section{AGRADECIMENTOS}

Os autores agradecem ao GIAQA- Grupo de Instrumentação e Automação em Química Analítica da Unicamp pelo apoio ao desenvolvimento deste estudo. São gratos ainda às agências de fomento CNPq e CAPES pelo apoio financeiro.

\section{REFERÊNCIAS}

1. Pessis, A. M.; Guidon, N.; World Archaeology 2007, 39, 406.

2. Hennetier, L.; Almeida, J. V.; Correia, A. M. S.; Ferreira, V. M.; Br. Ceramic Trans. 2001, 100, 72.

3. Čukovska, L.; Grupče, O.; Minčeva-Šukarova, B.; Kuzmanovski, I.; J. Brazil Chem. Soc. 2009, 20, 57.

4. Lopez-Acevedo, V.; Viedma, C.; Gonzalez, V.; Iglesia, A.; J. Cryst. Growth 1997, 182, 103.
5. Backbier, L.; Rousseau, J.; Anal. Chim. Acta 1993, 283, 855.

6. Menezes, R. R.; Ferreira, H. S.; Neves, G. A.; Ferreira, H. C.; Ceramica 2006, 52, 37.

7. Madariaga, J. M.; Maguregui, M.; Vallejuelo, S. F.; Knuutinen, U.; Castro, K.; Martinez-Arkarazo, I.; Giakoumaki, A.; Pitarch, A.; J. Raman Spectrosc. 2014, 45, 1059.

8. Kramar, S.; Urosevic, M.; Pristacz, H.; Mirti, B.; J. Raman Spectrosc. 2010, 41, 1441 .

9. Alberghina, M. F.; Barraco, R.; Brai, M.; Casaletto, M. P.; Ingo, G. M.; Marrale, M.; Policarpo, D.; Schillaci, T.; Tranchina, L.; Appl. Phys. A 2010, 100, 953.

10. Edwars, H. G. M.; Farwell, D. W.; Daffner, L.; Spectrochim. Acta, Part A 1996, 52, 1639.

11. Edwars, H. G. M.; Edwards, K. A. E.; Farwell, D. W.; Lewis, I. R.; Seaward, M. R. D. J.; J. Raman Spectrosc. 1994, 25, 99.

12. Aguayo, T.; Clavijo, E.; Eisner, F.; Ossa-Izquierdo, C.; Campos-Vallette, M. M.; J. Raman Spectrosc. 2011, 42, 2143.

13. Iordanidisa, A.; Garcia-Guinea, J.; Strati, A.; Gkimourtzinac, A.; Papoulidou, A.; Spectrochim. Acta, Part A 2011, 78, 874.

14. Garcia-Guinea, J.; Recio-Vazque, L.; Almendros, G.; Benavente, D.; Correcher, V.; Perez-Garcia, A.; Sanchez-Moral, S.; Fernandez-Cortes, A.; Environ. Earth Sci. 2013, 69, 1733.

15. Arroyo, F.; Villegas-Sánchez, R.; J. Cult. Heritage 2013, 14S, 109.

16. Paterakis, A. B.; Steiger, M.; Stud. Conserv. 2015, 60, 172.

17. Broggi, A.; Petrucci, E.; Bracciale, M. P.; Santarelli, M. L.; J. Raman Spectrosc. 2012, 43, 1560.

18. Dei, L.; Mauro, M.; Bitossi, G.; Thermochim. Acta 1998, 317, 133.

19. Bartl, B.; Havlínc, J.; Trejbal, J.; Durovi, M.; Thermochim. Acta 2013, 566, 292.

20. Kinoshita, A.; Mayer, E.; Mendes, V. R.; Figueiredo, A. M. G.; Baffa, O.; Radiat. Prot. Dosim. 2014, 159, 212.

21. Fontugne, M.; Shao, Q.; Frank, N.; Thil, F.; Guidon, N.; Boeda, E.; Radiocarbon 2013, 55, 1191.

22. Lage, M. C. S. M.; Revista de Geologia 1996, 9, 83.

23. Choay, F. Alegoria do Patrimônio, Unesp: São Paulo, 2001.

24. Alencar, W. J.; Santos, F. E. P.; Cisneros, J. C.; Silva, J. H.; Freire, P. T. C.; Viana, B. C.; Spectrochim. Acta, Part A 2015, 135, 1052.

25. Saikia, B. J.; Parthasarathy, G.; Sarmah, N. C.; Bull. Mater. Sci. 2008, 31, 775 .

26. Faria, D. L. A.; Lopes, F. N.; Souza, L. A. C.; Branco, H. D. O. C.; Quim. Nova 2011, 34, 1358.

27. Tourneret, L.; Berger, F.; Mavon, C.; Chambaudet, A.; Appl. Clay Sci. 1999, 14, 299.

28. Arrizabalaga, I.; Gómez-Laserna, O.; Carrero, J. A.; Bustamante, J.; Azibar Rodríguez, A.; Arana, G.; Madariaga, J. M.; Anal. Methods 2015, 7, 1061.

29. Santos, M. L.; Florentino, A. O.; Saeki, M. J.; Aparecida, A. H.; Fook, M. V. L.; Guastaldi, A. C.; Ecletica Quim. 2005, 30, 29.

30. Vratny, F.; Appl. Spectros. 1959, 13, 59.

31. Al-Hosney, H. A.; Grassian, V. H.; Chem. Phys. 2005, 7, 1266. 